\title{
Bias in conceptual priming
}

\author{
ANJALI THAPAR \\ Bryn Mawr College, Bryn Mawr, Pennsylvania \\ and \\ JEFFREY N. ROUDER \\ University of Missouri, Columbia, Missouri
}

\begin{abstract}
In recent years, Ratcliff, McKoon, and colleagues have argued that priming in perceptual implicit memory tests is the result of biases in information processing. Three experiments are presented that extend this framework to the conceptual implicit memory domain. Participants studied a list of words before receiving a set of general knowledge questions. For some questions, participants studied the correct answer; for others, they studied a similar but incorrect answer. Although study of a correct answer facilitated performance, study of the similar alternative hurt performance. Costs and benefits of previous study were observed in both production and forced-choice tasks. However, there was no benefit of previous study when participants studied both the correct answer and the similar but incorrect alternative. The pattern of results indicates that participants were biased to respond with previously studied words on the conceptual implicit memory test. This pattern is concordant with the biased information-processing approach to priming.
\end{abstract}

In recent years, there has been a debate in the memory literature on the nature of the mechanisms subserving priming on implicit memory tests. A standard view advanced by memory researchers is that priming reflects facilitation in the processing of a stimulus because of prior exposure to the stimulus in the experiment. The observed dissociations in performance on explicit and implicit memory tests have traditionally been viewed to reflect either different memory systems or the different types of processing activities engaged by the tests. The multiple memory systems (MMS) view postulates that dissociations between performance on implicit and explicit tests reflect storage of separate memory representations in two neuroanatomically and functionally distinct memory systems (Schacter, 1990, 1992, 1994; Squire, Knowlton, \& Musen, 1993; Tulving \& Schacter, 1990). Alternatively, the transfer appropriate processing (TAP) account assumes that performance on a memory test is influenced by overlap in the cognitive operations used during encoding and retrieval (Roediger, 1990; Roediger \& McDermott, 1993). TAP predicts dissociations between performance on explicit and implicit tests to the extent that these tests engage different types of en-

This research was supported in part by funds from the National Institutes of Mental Health (Grant 1 R03 MH 57702-01) and by funds from the National Institutes of Aging (Grant AG17083) to A.T. and by the National Science Foundation Grant BCS-9817561 to J.N.R. Correspondence concerning this manuscript should be addressed to A. Thapar, Department of Psychology, Bryn Mawr College, 101 N. Merion Ave., Bryn Mawr, PA 19010 (e-mail: athapar@brynmawr.edu) or J. N. Rouder, 210 McAlester Hall, University of Missouri, Columbia, MO 65211 (e-mail: jeff@banta.psyc.missouri.edu). coding and retrieval processes. Although the MMS and TAP accounts differ in their explanation for the nature of the distinction between implicit and explicit memory tests, both accounts assume that the effect of priming on a stimulus is beneficial. Prior exposure to a stimulus facilitates subsequent stimulus identification by enabling the person to perceive the stimulus more quickly and/or more accurately on subsequent trials.

More recently, an alternative view of priming based on the notion of bias has been advanced (Ratcliff \& McKoon, 1996, 1997; Rouder, Ratcliff, \& McKoon, 2000; see Morton, 1969,1970 , for an earlier presentation of the bias approach). The bias view postulates that the effect of previous study on memory is not to enhance the processing of a stimulus by changing representations or by adding new representations to an implicit memory system. Rather, prior exposure biases the processing of subsequent stimuli at test.

In a standard perceptual implicit memory test (e.g., word-fragment completion or word identification test), targets are either previously studied or new. Priming is observed when there is an advantage in the correct identification of previously studied targets relative to nonstudied targets. In the modified version of these tasks, Ratcliff and McKoon created an additional study condition for each target; items perceptually similar to the target were also studied. For example, in their 1996 study, the four-letter word died was presented at test and then masked. Participants studied died (study-target condition), lied (study-similar condition), or neither died nor lied (the no-study condition). Ratcliff and McKoon observed that previous study of the target facilitated performance but that previous study of the alternative hin- 
dered performance. Participants tended to produce the studied word at test regardless of the target stimulus. Ratcliff and colleagues went on to test the robustness of this cost-benefit pattern of priming on a variety of tasks (forced choice, naming, fragment and stem completion, and yes/no identification).

A crucial feature of the forced-choice task data is that this pattern of costs and benefits was obtained only when the alternatives at test were visually similar (e.g., died vs. lied). There was no effect of study when the alternatives were visually dissimilar (e.g., died vs. sofa). This pattern of benefits and costs for similar stimuli, but not for dissimilar stimuli, holds for spoken words embedded in noise (Ratcliff, Albritton, \& McKoon, 1997) and for object identification (Rouder et al., 2000). These findings pose a serious challenge to conventional theories of priming (such as MMS and TAP), which assume that prior study facilitates perceptual encoding of a test stimulus. These accounts do not predict costs in performance owing to prior study, nor do they predict that the mnemonic benefit of study depends on the similarity of the alternatives at test (Bowers, 1999; Ratcliff \& McKoon, 1997). In addition, the fact that prior study does not lead to bias for dissimilar pairs constrains bias accounts of priming.

In a recent summary of the debate, Bowers (1999) argues that the main difference between conventional theories of priming and the bias account revolves around the role of previous exposure. Conventional theories of priming (i.e., MMS and TAP) assume that previous exposure influences the representation of an item. Previous exposure is believed to facilitate the processing of a test stimulus either by adding a new representation to the memory system or by modifying an earlier representation. On the other hand, according to the bias theory, prior exposure biases the subsequent evaluation of ambiguous information (such as a flashed target), rather than enhancing the perceptual processing of repeated stimuli. According to the bias account, incoming stimuli are more likely to be interpreted in such a way as to support the identification of studied items at the expense of unstudied items. We will further elaborate on the distinction between theories that postulate changes in representations and those that do not in the General Discussion section.

The purpose of the experiments presented here is to extend the bias framework to the conceptual implicit memory domain. Our goal is to investigate the mechanisms subserving priming in conceptual implicit memory tests. Although the distinction between perceptual and conceptual implicit memory tests is well supported, the bias framework has been applied primarily to perceptual implicit memory tests. Priming on perceptual implicit memory tests is sensitive to manipulations of the surface characteristics of the stimuli (e.g., Roediger \& Blaxton, 1987; Weldon \& Roediger, 1987; Weldon, Roediger, Beitel, \& Johnston, 1995) but relatively in- sensitive to conceptual manipulations (e.g., Graf, Squire, \& Mandler, 1984; Greene, 1986; Jacoby \& Dallas, 1981), whereas priming on conceptual implicit memory tests tend to display the opposite pattern (Blaxton, 1989; Hamann, 1990; Srinivas \& Roediger, 1990). In light of the dissociations observed between perceptual and conceptual implicit memory tests, our goal was to test whether bias in information processing exerts an influence on priming in a conceptual implicit memory test. The conceptual implicit memory test used to test the bias account was a modified version of the general knowledge test. The general knowledge test consists of a series of questions, such as "What type of doctor besides a gynecologist specializes in pregnancy and childbirth?"Participants are presented with a list of words prior to answering the general knowledge questions. In the conventional conceptual implicit test paradigm, participants see the correct answers to some of the questions in the study phase. In this test, evidence for priming is observed if participants produce more correct responses to questions for which the answers were previously presented, relative to the baseline condition. The procedure used in the experiments presented below is a modified version of the original general knowledge test. Each general knowledge question was assigned to one of three study conditions: The correct answer was studied (for the above question, the correct answer is obstetrician), a plausible incorrect alternative was studied (e.g., pediatrician), or neither the answer nor a plausible incorrect alternative was studied during the study phase. The dependent variable is the proportion of correct responses. The research questions assessed by this paradigm are straightforward: Is there systematic evidence for costs as well as benefits in priming on a conceptual memory test? Second, if patterns of costs and benefits are observed, are they consistent with the bias processing account that has been successful in the perceptual domain?

A partial answer to the questions above can be found in a paper by Kelley and Lindsay (1993). Kelley and Lindsay were interested in the effect of prior exposure on priming in conceptual implicit memory tasks. They hypothesized that previous study of an item would increase its overall familiarity and that this, in turn, would increase the likelihood that participants would subsequently produce the item as the response to a question on a general knowledge test. Kelley and Lindsay also thought that because previously studied words would appear more familiar, participants would have more confidence in their answers. In order to test these hypotheses, the authors included a condition in which subjects were exposed to both correct answers and semantically related but incorrect answers during the study phase. Previous exposure to the item increased the probability that the item was produced, confidence that the studied item was indeed the correct answer when it was produced, and the speed of production. This pattern of results was observed for both the correct and the semantically related incor- 
rect study conditions. Although Kelley and Lindsay were not explicitly testing a bias approach to priming on conceptual memory tests, the observed pattern of cost and benefit of prior exposure to performance is consistent with the bias explanation of priming.

\section{GENERAL METHOD}

In the three experiments presented below, we assessed the effects of previous study with the general knowledge test. Experiment 1 used a response production procedure to assess the effects of previous study on the general knowledge test. Experiment 2 used the standard forced-choice response procedure to assess the costs and benefits of previous study in the general knowledge test. Experiment 3 also used a forced-choice procedure. However, in this experiment, there were two study conditions. In the study-both condition, both the correct answer and the plausible alternative were studied. In the study-neither condition, neither the correct answer nor the plausible alternative was studied. Experiment 1 is a partial replication of Kelley and Lindsay (1993). Experiments 2 and 3 use a forced-choice procedure to provide more stringent tests of the biased processing account of conceptual priming.

\section{Materials}

One hundred general knowledge questions served as stimuli (Blaxton, 1989). Thirty of these questions served as the critical test questions; the remaining 70 served as fillers. Each of the critical test questions was assigned a correct answer and a plausible but incorrect alternative. The plausible but incorrect alternative reflected common errors made by a pilot group of 22 participants (pilot participants were only administered the questionnaire; they did not study any words previously). These stimuli were used for all three of the experiments reported here. (Copies of test materials are available upon request.)

\section{Procedure}

The participants were tested individually. The instructions in the study phase were to "Remember the words for a later memory test." Sixty words were presented on a computer monitor for $3.0 \mathrm{sec}$ each. Of the 60 words, 20 were critical words ( 10 words were correct answers, another 10 words were plausible alternatives); the remaining 40 were filler words. The 1 st and last 5 words on the study list were always filler words. This order was used to control against recency and primacy effects. Other than the 1st and last 5 words, the word order was randomized for each participant.

After the study phase, the participants were told that the purpose of the study was to investigate the effects of different types of intervening stimuli on memory for the words on the study list. The participants were told that participants were randomly assigned to the "math problem" condition or the "verbal problem" condition and that they had been assigned to the "verbal problem" condition. In addition, they were told that occasionally a word that had appeared during the study phase would appear as a possible answer but that it was not necessarily the correct answer. The participants were informed that half of the studied words were indeed wrong answers and that they should not respond with an alternative on the basis of its status as a previously studied word. These two instructions were used to help eliminate participants' tendencies to select the studied word as the answer when they were guessing. ${ }^{1}$ The participants were told that their memory for the words on the study list would be tested upon completion of the general knowledge test.

The general knowledge test immediately followed the study phase. The participants were instructed to read each test question. After reading the test question, the participants hit the space bar. In Experiment 1, the participants generated their responses to the questions. The first 5 questions were fillers, after which the order of the remaining fillers and questions was randomized for each participant. Each participant answered 10 questions in which the answer was studied, 10 questions in which the plausible alternative was studied, and 10 questions in which neither was studied. In Experiments 2 and 3 , the participants then received two potential oneword answers. The participants were instructed to hit the " $Z$ " key if the correct answer to the question was the first word option and the "?" key if the correct answer was the second word option. The location (left or right) of the correct response was counterbalanced across subjects. Questions were counterbalanced so that, for each question, all three study conditions occurred equally often. Upon completion of the general knowledge test, the participants were debriefed regarding the true nature of the test, and their questions were answered.

\section{EXPERIMENT 1}

In Experiment 1, we assessed the effects of previous study on the general knowledge test, using a response production procedure. The target was studied, a plausible incorrect answer was studied, or neither the target nor the plausible incorrect answer was studied. After reading the questions, the participants had to type in their response. They were allowed to type "Don't Know," indicating they could not produce a guess.

\section{Method}

Thirty Northwestern University students served as participants in partial fulfillment of an introductory class requirement.

\section{Results}

The results are displayed in Table 1. A one-factor repeated measure analysis of variance (ANOVA) revealed that the effect of study condition for the correct answer was significant $\left[F(2,58)=5.06, M S_{\mathrm{e}}=0.22, p<.01\right]$. There was a performance benefit from studying the correct answer; the probability of a correct response increased by .09 over the no-study baseline condition. However, this gain was offset by a performance cost from studying the plausible alternatives; the probability of a correct answer decreased by .08, as compared with the no-study baseline condition. A one-factor repeated measure ANOVA revealed that the effect of study condition for the alternative word was significant $[F(2,58)=$

Table 1

Probability of Response on General Knowledge Test (With Standard Errors)

\begin{tabular}{|c|c|c|c|c|c|c|c|c|}
\hline \multirow[b]{3}{*}{ Response } & \multicolumn{8}{|c|}{ Study Conditions } \\
\hline & \multicolumn{2}{|c|}{ Correct } & \multicolumn{2}{|c|}{ Plausible } & \multicolumn{2}{|c|}{ Neither } & \multicolumn{2}{|c|}{ Both } \\
\hline & $M$ & $S E$ & $M$ & $S E$ & $M$ & $S E$ & $M$ & $S E$ \\
\hline \multicolumn{9}{|l|}{ Experiment 1} \\
\hline Target & .41 & .05 & .24 & .05 & .32 & .03 & - & \\
\hline Alternative & .08 & .01 & .18 & .02 & .09 & .01 & - & \\
\hline Experiment 2 & & & & & & & & \\
\hline Target & .77 & .04 & .57 & .05 & .68 & .05 & - & - \\
\hline Experiment 3 & & & & & & & & \\
\hline Target & - & - & - & - & .64 & .02 & .63 & .02 \\
\hline
\end{tabular}


3.69, $\left.M S_{\mathrm{e}}=0.13, p<.05\right]$. When the participants studied plausible alternatives, they were more likely to give the alternative as the answer (.18) than they were in the baseline condition (.09) or in the study correct condition (.08). These results replicate Kelley and Lindsay's (1993) findings; previously studied words are more likely to be given as answers to a general knowledge test, even when they are incorrect. The results demonstrate that bias exerts a role in priming in a conceptual implicit memory test.

\section{EXPERIMENT 2}

The forced-choice response procedure has played an important role in assessing the various accounts of perceptual priming that have been proposed. However, to our knowledge, the forced-choice procedure has yet to be applied to the conceptual implicit memory domain. In Experiment 2, the costs and benefits of previous study in the general knowledge test were assessed with a forcedchoice response procedure. In the test phase, the participant had to choose between the correct answer and the plausible alternative. The participants had previously studied the correct answer, the plausible alternative, or neither the correct nor the plausible alternative.

\section{Method}

Thirty students from Bryn Mawr College served as participants in partial fulfillment of an introductory psychology class requirement.

\section{Results}

The results are displayed in Table 1 . A one-factor repeated measure ANOVA revealed that the effect of study condition was significant $\left[F(2,58)=5.39, M S_{\mathrm{e}}=0.32\right.$, $p<.01]$. There was a performance benefit from studying the correct answers; the probability of a correct response increased by .09 over the no-study baseline condition. This gain was offset by a performance cost from studying the plausible alternatives; the probability of a correct response decreased by .11, as compared with the nostudy baseline condition. These results support a bias interpretation of the data; previously studied words are more likely to be given as answers, even when they are incorrect. The results obtained in Experiment 2 corroborate the previously reported forced-choice results with perceptual implicit memory tests and demonstrate that bias in information processing contributes to priming in a conceptual implicit memory test.

\section{EXPERIMENT 3}

In Experiment 3, we again assessed the effects of prior study on the general knowledge test with a forced-choice procedure. As has been pointed out by Ratcliff and McKoon (1997), Bowers (1999), and Wagenmakers and colleagues (Wagenmakers, Zeelenberg, \& Raaijmakers, 2000; Zeelenberg, Wagenmakers, \& Raaijmakers, 2000), there is an additional forced-choice paradigm in which the biased processing approach and MMS and TAP make differential predictions. The two conditions in this paradigm are the study-both condition (both the correct answer and the plausible alternative are studied) and the study-neither condition (neither the correct answer nor the plausible alternative is studied). According to the biased processing framework, studying both alternatives is equivalent to studying neither of the alternatives, and there should be no difference in performance. However, if previous study leads to enhanced processing of the stimulus, performance should be better in the condition in which both alternatives are studied, relative to the condition in which neither alternative is studied.

\section{Method}

Twenty-eight students from Villanova University and 12 students from Bryn Mawr College served as participants. Students from both institutions participated to fulfill requirements in psychology courses. Each target was in one of two study conditions, study-both or study-neither. These conditions were presented in a within-list design and were counterbalanced across participants and questions.

\section{Results}

The results are displayed in Table 1 . The effect of study condition ( study-both $=.63$; study-neither $=.64$ ) was not significant $[t(39)=-.59$, n.s.]. The power of this manipulation to detect an effect of the size observed in Experiment 2 was estimated to be .93 . These results indicate that prior study does not result in an overall improvement in performance. The failure to observe priming in the study-both condition is difficult to reconcile with theories of priming that assume that prior exposure enhances overall performance. However, these results are predicted in the biased processing accounts of priming. The results obtained in Experiment 3 corroborate the previously reported forced-choice results that have been observed in similar study-both/study-neither manipulations with perceptual implicit memory tests (Masson \& MacLeod, 1996; Ratcliff \& McKoon, 1997). ${ }^{2}$

\section{GENERAL DISCUSSION}

The purpose of this article is to extend the bias approach of priming to the conceptual implicit memory test domain. The results from the three experiments reported in this paper are consistent with the biased processing accounts of priming. One serious limitation of the bias research reported to date is that it has focused on perceptual implicit memory tests. Our goal was to investigate the mechanisms subserving priming in conceptual implicit memory tests. Specifically, we wanted to investigate whether previous study would result in both costs and benefits on a conceptual implicit memory test.

The results of Experiment 1 (production) and Experiment 2 (forced choice) are consistent: Study of correct answers facilitated performance, whereas study of incorrect 
but plausible answers hindered performance. Experiment 3 revealed that performance was not enhanced when participants studied both the correct answer and the incorrect but plausible alternative, as compared with the baseline condition, in which neither alternative was studied. Overall, the pattern of results reported here can best be characterized as the product of biases in the information-processing system and is analogous to those obtained in perceptual-priming studies.

One of the advances in priming research has been a shift from studying priming per se to studying priming as a means of understanding psychological processing on a set of tasks. For example, in the word identification domain, researchers are using priming to test theories of word identification, rather than to test theories of priming (e.g., Bowers, 1999; Ratcliff \& McKoon, 1997; for an example in the object recognition domain, see Rouder et al., 2000). The approach used here to relate priming in conceptual memory tasks to theoretical mechanisms is to assume that priming affects the way in which information is evaluated at the decision stage of processing. One framework for discussing decision making across a number of tasks is that of activation accumulation in nodes or counters, such as in Morton's (1969) logogen model or McClelland and Rumelhart's (1981) interactive activation model. Each possible response has a node associated with it, and each of these nodes slowly accumulates activation through the course of a trial. A decision is made (and a response is produced) the first time the activation in one of the nodes is sufficiently high. ${ }^{3} \mathrm{~A}$ general knowledge question would invoke a retrieval process that results in an increase in activation levels in relevant nodes. For example, when the participant is asked, "What material is the Taj Majal constructed from?" activation in nodes corresponding to such concepts as "concrete," "granite," and "marble" will rise. If the participant knows that the correct answer is marble, the activation in this node should rise at a greater rate than that in the other nodes. Overall, it is assumed that activation is accumulated by a noisy process, so errors can occur.

There are a number of different approaches for integrating the effects of previous study in priming in a conceptual implicit memory test within such a framework. To account for both costs and benefits, one straightforward approach is to assume that previous study affects either the resting activation level or the response criteria (such as in Morton's, 1969, logogen model). For example, previous study of granite may raise the resting activation or lower the criterion for the granite node. When the question about the Taj Majal is presented, less activation is needed to produce a granite response than would have been needed if granite had not been studied. This approach can readily explain the data in all of the three experiments. But this explanation cannot explain an important result in perceptual priming, in which previous study affects only performance in forced-choice identification tests when the alternatives are similar but not dissimilar. ${ }^{4}$ The approach above would generally predict a bias toward previous studied items regardless of the similarity relationship of the alternatives.

To address the effect of the alternative similarity, Ratcliff and McKoon (1997) and Rouder et al. (2000) propose a different type of bias. They assume that previous study affects the rate at which nodes increase activation. If granite is studied, the rate of activation gain in the granite node is increased, while the rate of activation gain in other similar nodes (concrete, marble) decreases. The net effect is aptly described by the metaphor that the granite node is "stealing" activation from its similar neighbors. Ratcliff, McKoon, and their colleagues provide processing mechanisms such that the stealing is limited only to nodes corresponding to similar items and does not occur for dissimilar ones. In the case in which both the correct answer and the incorrect but similar alternative are studied, both items steal from each other, and the bias effects cancel out. Therefore, bias in rate of accumulation is also consistent with the data of the three experiments.

A similar manipulation is needed to differentiate between the two types of bias explanations in the conceptualpriming domain. However, this manipulation is difficult to test in the conceptual-priming domain. The chief problem is that if items in a forced choice are too dissimilar, performance approaches ceiling, and bias patterns (or the lack thereof) are obscured. For example, suppose a participant is asked about the composition of the Taj Majal and is presented with the dissimilar alternatives of marble and paper. The answer is far more obvious than it would be if the alternatives were marble and granite, and performance approaches ceiling. The procedural advantage gained in the perceptual domain is that researchers can independently shorten stimulus duration when alternatives are dissimilar, so that performance with similar and dissimilar alternatives can be approximately equated. In light of this constraint, we can conclude that the results in conceptual priming to date are consistent with both theories of bias, either that previous study biases the response criterion (or resting activation) of corresponding nodes or that previous study biases the rate of activation increase at the expense of that for similar alternatives.

Although the paradigm used here does not provide the ability to distinguish between the two types of bias explanations, it does allow us to rule out some explanations. A strict interpretation of the MMS approachmore specifically, the perceptual representational system (PRS) that has been proposed by Schacter $(1990,1992)$ to account for priming in perceptual implicit tests, would not be able to account for priming on conceptual tests. If the effect of study was to form an implicit trace in a PRS (Schacter, 1990, 1992), there should be very little effect of previous study. Additional perceptual ease with a particular answer is not too helpful in this task, given its 
conceptual nature (especially in Experiment 1, which used a free response production procedure). However, even if one assumed that a system analogous to PRS but designed for conceptual priming existed, this system would still have difficulty in explaining the pattern of costs and benefits observed in our data (just as PRS fails to predict the pattern of costs and benefits observed in perceptual-priming tests).

Our results also rule out the proposition that the effect of previous study is to affect the representation of the stimulus and its connections with related concepts, possibly as a result of spreading activation. For example, suppose that at some point, each participant learned that the Taj Majal is indeed made of marble. When "marble" is presented at study, there should be activation of both the marble node and the Taj Majal node, and the connection should be strengthened. This strengthening of the connection between Taj Majal and marble is a change in representation of the Taj Majal concept, because the participant now more strongly associates marble with the Taj Majal. But, in the condition in which granite is studied, there is no change in representations, because the Taj Majal is not made of granite. That is, there will be no such strengthening of the Taj Majal node and the granite node, because activation of the granite node at study does not activate the Taj Majal node. Hence, there should be no costs to previous study of incorrect but plausible alternatives, a prediction that is inconsistent with the reported data.

This last theory of connection strengths allows us to draw a distinction between theories in which previous study changes representation to those in which it does not. As has been mentioned, changes in connection strengths are changes in representation, because the connections denote the associations among various concepts. But the bias theories do not postulate changes in representations. Although previous study may result in stealing activation or changing response criteria, the basic relationship between a concept and other concepts is unchanged. Studying granite does not affect one's underlying belief in the composition of the Taj Majal, although it will allow the granite node to be more active when granite and marble are in competition. In terms of the framework outlined here, theories that postulate changes in representation would predict increased rates of accumulation for studied items, but not at the expense of unstudied but similar alternatives. There are differential predictions between stealing-of-activation theories and gain-in-activation (from changes in representations) theories for Experiment 3. As was mentioned before, stealing theories predict no net effect of studying both the correct alternative and the similar but incorrect alternative over baseline. But if study leads to increased activation gain, we would expect participants to be more accurate overall when both alternatives are studied then when neither is studied. As was mentioned before, in Experiment 3 , there were no net effects of study.
The framework outlined here is related to the one proposed by Kelley and Lindsay (1993). Their framework for explaining the effects of previous study on general knowledge retrieval is based on the concepts of familiarity and perceptual ease. According to Kelley and Lindsay, the easier an answer pops into mind, the more confidence the participant will have that the answer is indeed the correct one. The role of previous study is to boost the familiarity of studied items and, hence, the ease with which they pop into mind. Although we do not rely on such concepts as ease and familiarity, the spirit of the frameworks is similar. Previous study biases the overall saliency of a potential answer in addition to the participant's semantic knowledge.

\section{REFERENCES}

Audley, R. J., \& Pike, A. R. (1965). Some alternative stochastic models of choice. British Journal of Mathematical \& Statistical Psychology, 18, 207-225.

Blaxton, T. A. (1989). Investigating dissociations among memory measures: Support for a transfer appropriate processing framework. Journal of Experimental Psychology: Learning, Memory, \& Cognition, 15, 657-668.

Bowers, J. S. (1999). Priming is not all bias: Commentary on Ratcliff and McKoon (1997). Psychological Review, 106, 582-596.

Graf, P., Squire, L. R., \& MAndler, G. (1984). The information that amnesic patients do not forget. Journal of Experimental Psychology: Learning, Memory, \& Cognition, 10, 164-178.

Greene, R. L. (1986). Word stems as cues in recall and recognition tasks. Quarterly Journal of Experimental Psychology, 38A, 663-673.

Hamann, S. B. (1990). Level of processing effects in conceptually driven implicit tasks. Journal of Experimental Psychology: Learning, Memory, \& Cognition, 16, 970-977.

JACOBY, L. L., \& DALlas, M. (1981). On the relationship between autobiographical memory and perceptual learning. Journal of Experimental Psychology: General, 110, 306-340.

Kelley, C. M., \& Lindsay, D. S. (1993). Remembering mistaken for knowing: Ease of retrieval as a basis for confidence in answers to general knowledge questions. Journal of Memory \& Language, 32, 1-24.

Masson, M. E. J., \& MacLeod, C. M. (1996). Contributions of processing fluency to repetition effects in masked word identification. Canadian Journal of Experimental Psychology, 50, 9-21.

McClelland, J. L., \& Rumelhart, D. E. (1981). An interactive activation model of context effects in letter perception: Part 1. An account of basic findings. Psychological Review, 88, 375-407.

McKoon, G., \& Ratcliff, R. (2001). Counter model for word identification: Reply to Bowers (1999). Psychological Review, 108, 674-681.

Morton, J. (1969). The interaction of information in word recognition. Psychological Review, 76, 165-178.

Morton, J. (1970). A functional model of memory. In D. A. Norman (Ed.), Models of human memory (pp. 203-254). New York: Academic Press.

PIKE, R. (1973). Response latency models for signal detection. Psychological Review, 80, 53-68.

Ratcliff, R., Albritton, D., \& McKoon, G. (1997). Bias in auditory priming. Journal of Experimental Psychology: Learning, Memory, \& Cognition, 23, 143-152.

Ratcliff, R., \& McKoon, G. (1996). Bias effects in implicit memory tasks. Journal of Experimental Psychology: General, 125, 403-421.

Ratcliff, R., \& McKoon, G. (1997). A counter model for implicit priming in perceptual word identification. Psychological Review, 104, 319-343.

Roediger, H. L., III (1990). Implicit memory: Retention without remembering. American Psychologist, 45, 1043-1056.

Roediger, H. L., III, \& Blaxton, T. A. (1987). Effects of varying 
modality, surface features, and retention interval on priming in wordfragment completion. Memory \& Cognition, 15, 379-388.

Roediger, H. L., III, \& McDermott, K. B. (1993). Implicit memory in normal human subjects. In F. Boller \& J. Grafman (Eds.), The handbook of neuropsychology (Vol. 8, pp. 63-131). New York: Elsevier.

Rouder, J. N., Ratcliff, R, \& McKoon, G. (2000). A neural network model of implicit memory for object recognition. Psychological Science, 11, 13-19.

SCHACTER, D. L. (1990). Perceptual representation systems and implicit memory: Toward a resolution of the multiple memory systems debate. In A. Diamond (Ed.), Development and neural bases of higher cognitive functions. (Annals of the New York Academy of Sciences, Vol. 608, pp. 543-572). New York: New York Academy of Sciences.

Schacter, D. L. (1992). Priming and multiple memory systems: Perceptual mechanisms of implicit memory. Journal of Cognitive Neuroscience, 4, 244-256.

Schacter, D. L. (1994). Priming and multiple memory system: Perceptual mechanisms of implicit memory. In D. L. Schacter and E. Tulving (Eds.), Memory systems 1994 (pp. 233-268). Cambridge, MA: MIT Press.

Squire, L. R., Knowlton, B., \& Musen, G. (1993). The structure and organization of memory. Annual Review of Psychology, 44, 453-495.

Srinivas, K., \& Roediger, H. L., III (1990). Classifying implicit memory tests: Category association and anagram solution. Journal of Memory \& Language, 29, 389-412.

Tulving, E., \& Schacter, D. L. (1990). Priming and human memory systems. Science, 247, 301-306.

Wagenmakers, E.-J. M., Zeelenberg, R., \& RaAijmakers, J. G. W. (2000). Testing the counter model for perceptual identification: Effects of repetition priming and word frequency. Psychonomic Bulletin \& Review, 7, 662-667.

Weldon, M. S., \& Roediger, H. L., III (1987). Altering retrieval demands reverses the picture superiority effect. Memory \& Cognition, 15, 269-280.

Weldon, M. S., Roediger, H. L., III, Beitel, D. A., \& Johnston, T. R. (1995). Perceptual and conceptual processes in implicit and explicit tests with picture fragment and word fragment cues. Journal of Memory \& Language, 34, 268-285.

Zeelenberg, R., Wagenmakers, E.-J. M., \& RaAijmakers, J. G. W.
(2000). Repetition priming in implicit memory tasks: Prior study causes enhanced discriminability, not just bias. Manuscript submitted for publication.

\section{NOTES}

1. McKoon and Ratcliff (2001) have stressed the importance of giving specific instruction to avoid having participants follow a conscious strategy of responding with the studied items on the test. In addition, Kelley and Lindsay (1993) demonstrated that the effect observed in their data was not based on intentional retrieval of the studied items (Experiments 2 and 3 ) or on explicit recognition of the answer as having been previously presented on the study list (Experiment 4a).

2. Recently, the generality of the lack of priming in the studied-both condition has been challenged. An improvement in performance as a result of studying both alternatives has been found under very specific circumstances (the improvement has generally been found only for lowfrequency items after multiple study attempts).

3. There are two different decision rules that have been adopted in practice. One common rule is to assume that each node has its own criterion and that a response is produced the first instance some node's activation exceeds its criterion (e.g., Audley \& Pike, 1965; Morton, 1969). The other rule is to assume that a response is produced when the activity in the most active node exceeds the activity in the other nodes by a criterial amount (e.g., Pike,1973; Ratcliff \& McKoon, 1997; Rouder et al., 2000).

4. Recently, the generality of the finding that previous study affects performance with similar alternatives, but not with dissimilar alternatives, has been challenged. Bowers (1999) reports bias for dissimilar alternatives in word identification with quickly flashed words that were subsequently masked. However, McKoon and Ratcliff (2001) contend that the instructions used by Bowers were flawed. According to McKoon and Ratcliff, the participants in Bowers's experiments were guessing with words from the study list via explicit memory when they failed to detect the quickly flashed word. In sum, the finding of no effect of previous study when the alternatives are dissimilar, as well as the challenge, is still controversial.

(Manuscript received June 15, 2000; revision accepted for publication April 11, 2001.) 